\title{
Anaphase-Promoting Complex 7 is a Prognostic Factor in Human Colorectal Cancer
}

\author{
Ik Yong Kim, Hye Yeon Kwon, Kwang Hwa Park ${ }^{1}$, Dae Sung Kim \\ Departments of Surgery and ${ }^{1}$ Pathology, Yonsei University Wonju College of Medicine, Wonju, Korea
}

Purpose: The anaphase-promoting complex (APC) is a multiprotein complex with E3 ubiquitin ligase activity and is required for ubiquitination of securin and cyclin-B. Several APC-targeting molecules are reported to be oncogenes. Dysregulation of APC may be associated with tumorigenesis. This study examines the relationship between APC expression and clinicopathological factors and evaluates the possibility of an aberrant APC function in colorectal carcinomas (CRCs).

Methods: To determine whether the loss of APC7 expression is related to tumorigenesis, we used tissue micro-arrays in 114 resected CRCs to scrutinize the expressions of APC7 and Ki-67 immunohistochemistry and to find relations with clinocopathologic parameters. The expression of APC7 was defined as positive for summed scores of staining intensities from 0 to $3+$.

Results: Forty-four cases (67.7\%) of colon cancer and 38 cases (77.6\%) of rectal cancer showed immunopositive reactions to APC. The grade of APC expression was not statistically correlated with tumor location, age, T or TNM stage, or differentiation. However, the expression of APC did correlate with the expression of Ki-67 and to the tumor recurrent. Higher APC expression showed the better 5 -year overall survival rate in $74 \%$ of grades 2,3 groups (high expression) than $57 \%$ of grades 0,1 groups (lower expression) respectively $(\mathrm{P}=0.042)$.

Conclusion: Positive APC expression may be a good prognostic factor for patients with CRC, and the loss of APC expression in tumor tissue may be related with the risk for recurrence and a poor survival rate compared to high APC expression. Further study of APC in controlling the cell cycle as aberrant function in CRC is needed.

\section{Keywords: Anaphase-promoting complex-cyclosome; Adenocarcinoma; Colorectal neoplasms; Cell cycle}

\section{INTRODUCTION}

The anaphase-promoting complex (APC) is an E3 ubiquitin ligase that controls mitotic progression $[1,2]$. APC is a polymeric protein complex composed of at least 11 subunits and contains tetratricopeptide repeat proteins (APC3, 5, 6, 7, and 8), a cullin homolog (APC2), and a ring-H2 finger domain (APC11). APC requires two WD40 repeat-containing coactivators, $\mathrm{Cdc} 20$ and

Received: October 6, 2016 - Accepted: May 13, 2017

Correspondence to: Dae Sung Kim, M.D.

Department of Surgery, Yonsei University Wonju College of Medicine,

20 Ilsan-ro, Wonju 26426, Korea

Tel: +82-33-741-0573, Fax: +82-33-741-0574

E-mail: daeskim@yonsei.ac.kr

(C) 2017 The Korean Society of Coloproctology

This is an open-access article distributed under the terms of the Creative Commons Attribution NonCommercial License (http://creativecommons.org/licenses/by-nc/4.0) which permits unrestricted noncommercial use, distribution, and reproduction in any medium, provided the original work is properly cited.
Cdh1, to recruit and select various substrates at different stages of the cell cycle, and APC3 and APC7 were recently suggested to interact with these APC activators [3]. Genetic alterations, typically associated with malignant cell phenotypes, affect genes involved in DNA repair and apoptosis, cell adhesion and invasion, angiogenesis, and finally, cell proliferation and cell cycle control [4].

Chromosome instability is believed to contribute to malignant transformation because the majority of malignant human cancers exhibit chromosomal gain or loss [5] and because mitotic defects, including chromosome aberrations, are frequently found in malignant cancers [6-8]. Because of the roles played by APC in mitotic cell-cycle progression, the timely activation of APC is thought to be important for maintaining accurate chromosome separation. In addition, a report indicating that the mitotic spindle checkpoint was reached by preventing APC activation [9]. It was also suggested that the dysregulation of APC may give rise to abnormal chromosome segregation, resulting in aneuploidy.

Several APC-targeting molecules, such as securin, polo-like ki- 
nase, aurora kinase, and SnoN, have been reported to be oncogenes. Dysregulation of APC may be associated with tumorigenesis, so one can hypothesize that the abnormal regulation of APC may be involved in malignant transformation through chromosome instability [10]. However, the clinical significance and the involvement of APC in tumorigenesis have not yet been investigated. In a previous study, we investigated immunohistochemically the levels of APC7 in various cancer tissues and found weak APC7 expression in colorectal adenocarcinomas [11]. This study examines the relationship between APC expression and the clinicopathological variables in an attempt to determine the role of the APC in colorectal cancer and to evaluate the possibility of an aberrant APC function in surgically-resected adenocarcinomas of the colorectum. Therefore, we investigated the expression of APC7 in 114 colorectal carcinomas and examined the relationship between the expression of APC7 and the clinicopathologic parameters.

\section{METHODS}

We retrospectively studied patients $(\mathrm{n}=114)$ who presented at the Department of Surgery, Yonsei University, Wonju Severance Christian Hospital from January 2000 to December 2002 for treatment of an adenocarcinoma of the colorectum. Curative surgery, reviews of the pathology reports and clinical charts of the patients, age at surgery, gender, tumor location, Dukes stage, and differentiation were investigated in the short-term and the longterm survivors. Tumors were graded according to the TNM classification. Each adenocarcinoma was staged according to the standards of the American Joint Committee on Cancer (7th edition AJCC, 2010). Histological diagnoses were established based on standard hematoxylin and eosin (H\&E)-stained sections according to the guidelines of the World Health Organization (WHO). The details on the distributions of clinicopathological factors in the study cohort are listed in Table 1.
Polyclonal antibodies against mouse APC7 were raised in New Zealand white (NZW) rabbits by immunization with recombinant APC7 protein. Briefly, recombinant mouse APC7 proteins were produced in Escherichia coli by using a pET32 expression vector system (Novagen, Madison, WI, USA). The resulting 6x histidine-tagged APC7 proteins were purified by using Ni-NTA affinity chromatography (Qiagen, Hilden, Germany). A NZW rabbit was then immunized with the purified APC7 protein and boosted twice. Blood was collected from the auricular artery, and serum was prepared by clotting and differential centrifugal separation (10,000 g for 10 minutes). APC7-specific antibodies were further purified by binding serum to APC7-coupled nitrocellulose and eluting with $100 \mathrm{mmol} / \mathrm{L}$ glycine- $\mathrm{HCl}$ buffer $(\mathrm{pH}, 2.5)$.

For the tissue micro-array (TMA) construction, the areas of the tumors were first identified on H\&E-stained slides. The areas with hemorrhage, necrosis, and histological artifacts were excluded. The selected areas were sampled from the paraffin block by using 5 -mm-sized tip punches and were re-embedded in a TMA mold with 20 cores per block (Quick Ray, Unitma, Seoul, Korea) (Fig. 1). Through the use of a microtome, TMA blocks were cut into 4- $\mu \mathrm{m}$ slices for immunohistochemical staining. An $\mathrm{H} \& \mathrm{E}$ stain was performed on each block of the tissue array to confirm the presence of cancer in the tissue cores.

The immunohistochemical technique was used to detect Ki-67 and APC (ChemMate Envision Kit; K5007, DAKO, Glostrup, Denmark). Paraffin-embedded tissue array sections of $4 \mu \mathrm{m}$ in thickness were de-paraffinized with xylene and dehydrated gradually with graded alcohol. For antigen retrieval, tissue sections were boiled in Tris ethylenediaminetetraacetic acid (EDTA) buffer $(\mathrm{pH}, 9.0) 3$ times at $100^{\circ} \mathrm{C}$ for 5 minutes in a microwave oven and then cooled for 20 minutes at room temperature. Soaking the sections in 3\% hydrogen peroxidase for 5 minutes blocked endogenous peroxidase activity. After the slides had been washed in Tri Buffered Saline (TBS, S3001, DAKO) for 10 minutes, they were incubated with primary antibodies (1:50 dilution) overnight in a

Table 1. Expression of APC7 in various human tissues

\begin{tabular}{|c|c|c|}
\hline \multirow{2}{*}{ Expression of $\mathrm{APC} 7^{\mathrm{a}}$} & \multicolumn{2}{|r|}{ Tissue type } \\
\hline & Normal tissue & Tumor tissue \\
\hline Negative & $\begin{array}{l}\text { Adipocytes, brain, hepatocytes, skeletal muscle cells, spi- } \\
\text { nal cord }\end{array}$ & $\begin{array}{l}\text { Lipoma, pleomorphic adenoma of salivary gland, low grade urothelial carci- } \\
\text { noma, chondrosarcoma, adenoid cystic carcinoma, renal cell carcinoma, } \\
\text { high grade ductal carcinoma of breast }\end{array}$ \\
\hline Positive & $\begin{array}{l}\text { Basal cells of epidermis, bronchial epithelium, ductal cells } \\
\text { of breast, ductal cells of pancreas, ductal cells of sali- } \\
\text { vary glands, endometrial glands, kidney epithelium, } \\
\text { gastric mucosa, prostate glands and ducts, urothelial } \\
\text { epithelium, fibroblasts, germinal center cells }\end{array}$ & $\begin{array}{l}\text { Adenocarcinoma of colon, adenocarcinoma of endometrium, adenocarcinoma } \\
\text { of pancreas, adenocarcinoma of prostate, adenocarcinoma of stomach, } \\
\text { ductal carcinoma of pancreas, hepatocellular carcinoma, high grade uro- } \\
\text { thelial carcinoma, papillary serous carcinoma of ovary, squamous cell car- } \\
\text { cinoma of esophagus, squamous carcinoma of cervix, leiomyosarcoma of } \\
\text { uterus, malignant lymphoma, melanoma, seminoma of testis }\end{array}$ \\
\hline
\end{tabular}

aImmunohistochemical data from tissue arrays (Tissue-Array Co., Seoul, Korea) stained with anti-anaphase-promoting complex (APC)7 antibodies. Tissue-array slides mounted with 50 normal or 50 tumor cores contained 17 normal or 22 tumor tissues, respectively, in triplicate or duplicate. Negative APC7 expression is represented by staining intensities of 0 or $1+$ whereas positive expression is represented by staining intensities of $2+$ or $3+$, as designated in Fig. 2A. Average staining intensities of several cores were used to determine tissue expression. Adapted from Park et al., Breast Cancer Res 2005;7:238-47 [11]. 
Volume 33, Number 4, 2017

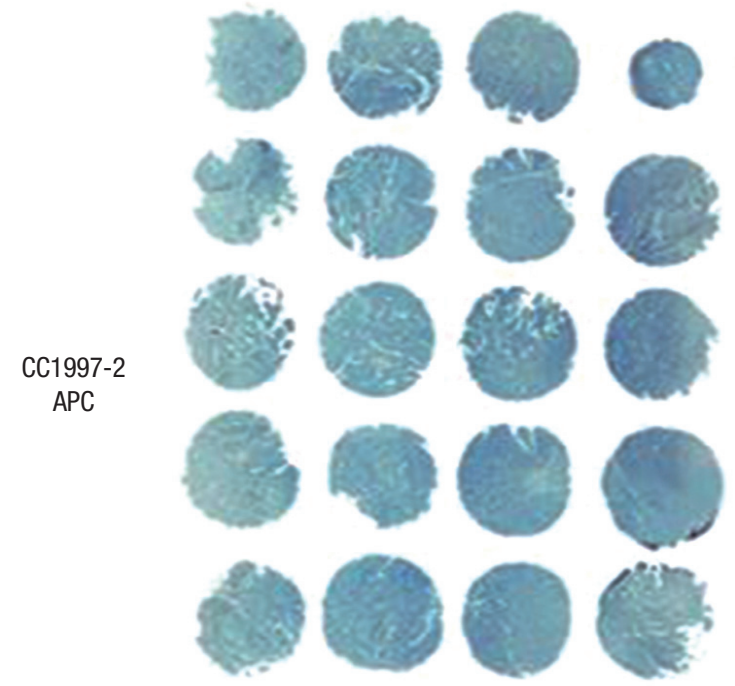

Fig. 1. Photomicrograph showing a slide of immunohistochemical staining of $4-\mu \mathrm{m}$-thick sections obtained from the tissue array block. wet incubation box. After the slides had been washed in TBS for 10 minutes again, they were incubated with dextran coupled with peroxidase and secondary antibodies for 30 minutes in the wet incubation box. The slides were washed for 10 minutes again with TBS and then incubated with substrate chromogen solution for 10 minutes. Lastly, the slides were washed with distilled water and were then briefly counterstained with hematoxylin and mounted.

The monoclonal antibody of APC and rabbit anti-human Ki-67 (DAKO) were used at 1:50 dilutions. We performed immunohistochemical staining by using Cap-plus detection kits (Zymed Laboratories, South San Francisco, CA, USA). For antigen retrieval, Tris-EDTA buffer $(\mathrm{pH}, 8.0)$ for the retinoblastoma protein $(\mathrm{pRb})$ and sodium citrate buffer $(\mathrm{pH}, 6.0)$ for Ki-67 were used. The staining procedure was similar to that with the ChemMate Envision detection kit. The grading of the immunohistochemical results was performed without the knowledge of the clinicopathologic details. In order to evaluate the immunohistochemical staining for APC and Ki-67 antigen, we divided the staining results into four degrees from 0 to 3 points. For APC, every tumor was
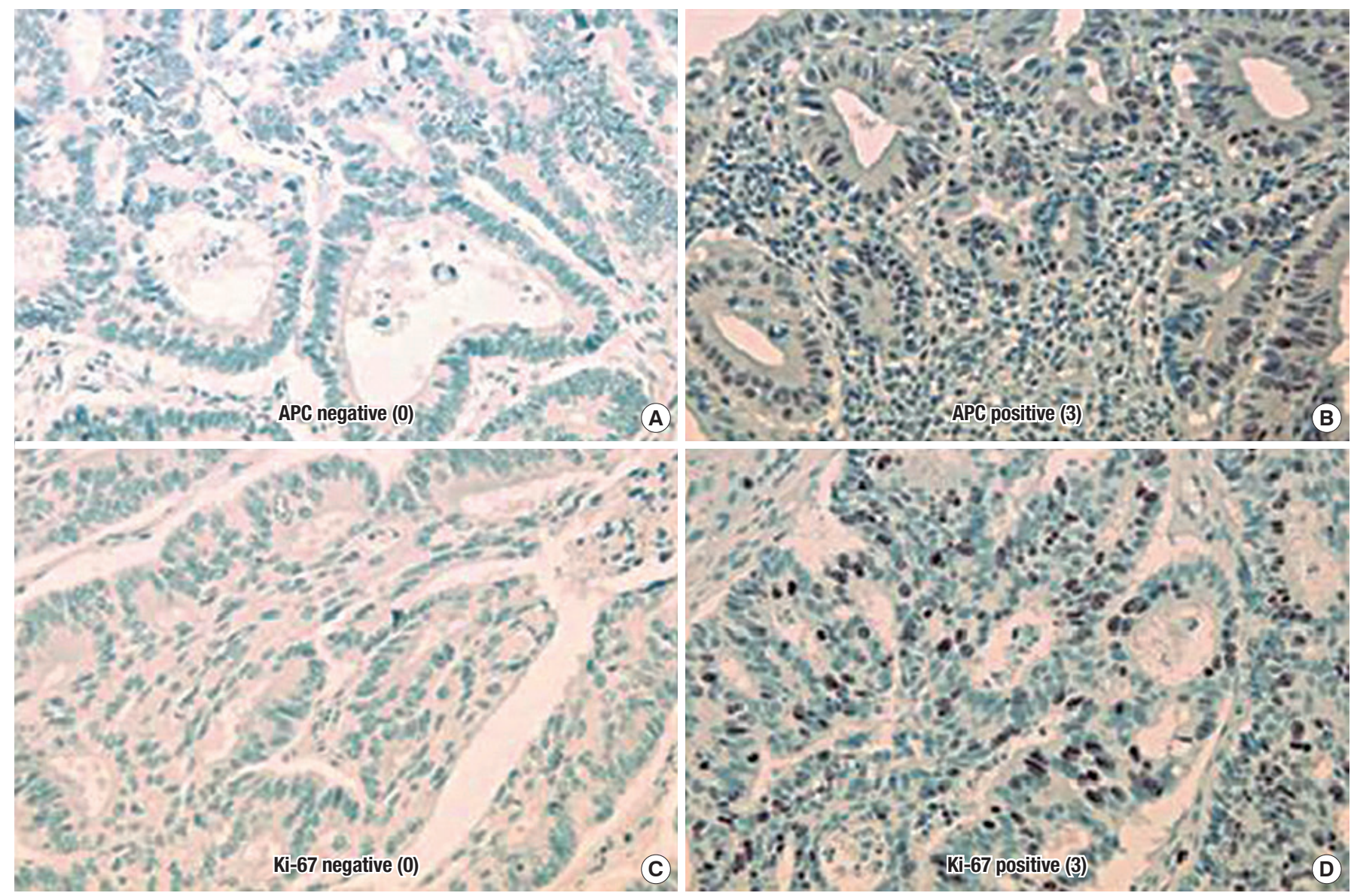

Fig. 2. Photomicrographs showing the immunohistochemical stains: (A) the nucleus of the tumor cell is negative for anaphase-promoting complex (APC) ( $\times 400)$, (B) APC expression was strong in the nucleus $(\times 400)$, (C) the tumor cell is negative for Ki-67 ( $\times 400)$, and (D) Ki-67 expression was strong in the nucleus $(\times 400)$. 
given a score for the intensity of the nuclei staining (no staining, 0 ; low staining, 1; medium staining, 2; strong staining, 3). The Ki-67 labeling index was defined as the percentage of positively stained cells in five high-power fields $(\times 400)$. At least 1,000 cells per field were counted. The immunohistochemical scoring for Ki-67 antigen was determined as follows: 3 points were assigned if the ratio of positively-stained nuclei was $50 \%$ or more, 2 points if the ratio was between $25 \%$ and $49 \%, 1$ point if it was between 1 and $24 \%$, and 0 point if it was negative (Fig. 2).

In the statistical analysis, P-values less than 0.05 were considered statistically significant. The life table method was used for recurrence, stage, and APC and Ki-67 expressions. A SPSS ver. 11.5 (SPSS Inc., Chicago, IL, USA) Kaplan-Meier survival analysis was used to determine the correlation between the overall survival and grade of APC expression.

\section{RESULTS}

To search for differentially expressed APC7 in normal and cancerous tissues, we performed immunohistochemical analyses with purified mouse APC7 antibodies by using tissue array slides containing 50 normal or 50 tumor tissue cores. We compared the APC7 expressions of the cores by assessing the averaged staining intensities ( 0 to $3+$ ). Staining of $\geq 2+$ was defined as positive expression and that of $\leq 1+$ as negative expression. Table 1 lists the APC7 expressions of 17 normal and 22 tumor tissues with multiple cores. Positive staining was observed in rapidly growing, normal epithelial tissues [11]. In contrast, slow growing, but more differentiated, tissues, such as skeletal muscle tissue, adipocytes, spinal cord and brain tissues, and basal stromal tissues near epithelial cells, exhibited no or weak immune reactivity to APC7. In addition, slowly growing tumors, such as chondrosarcomas, lipomas, low-grade urothelial carcinomas, and renal cell carcinomas, tended to show weak reactivity to APC7 whereas most tumor tissues with high proliferation rates were positive. Interestingly, some ductal carcinomas of the breast with an undifferentiated, high histologic grade exhibited weak reactivity to APC7.

To establish the relationship between the clinicopathologic parameters and APC7 expression, we analyzed 114 cases of colon cancer. Follow-up duration was more than 5 years. Eighty patients could be followed, and recurrence occurred in 32 patients. The clinicopathologic characteristics of the cases analyzed in this study are shown in Table 2. In all rectal cancers, carcinoma cells could be observed under a light microscope. We histologically classified the tumors according to the WHO classification and found 103 cases of adenocarcinomas ( 2 well-differentiated, 98 moderately differentiated, 3 poorly differentiated), 10 mucinous adenocarcinomas, and 1 signet-ring cell carcinoma. The median age of the patients was $58.3 \pm 13.1$ years (range, $15-82$ years). The tumor was located at the colon and the rectum in $65(57.02 \%)$ and 49 cases (42.98\%), respectively. Of the tumors, $0,20,88$, and 6 were categorized as T stages 1, 2, 3, and 4, respectively (Table 2).
Table 2. Patients' clinical and pathological characteristics of the whole sample and their associations between the 2 treatment subgroups

\begin{tabular}{|c|c|c|c|c|c|c|c|}
\hline \multirow{3}{*}{ Variable } & \multirow{3}{*}{$\begin{array}{c}\text { No. of } \\
\text { cases } \\
\text { (\% of total) }\end{array}$} & \multicolumn{5}{|c|}{ APC expression IHC grade } & \multirow{3}{*}{ P-value } \\
\hline & & \multirow{2}{*}{$\frac{\text { Negative }}{0}$} & \multicolumn{4}{|c|}{ Positive } & \\
\hline & & & 1 & 2 & 3 & All & \\
\hline Location & & & & & & & 0.256 \\
\hline Colon & $65(57.0)$ & $21(32.3)$ & 16 & 15 & 13 & $44(67.7)$ & \\
\hline Rectum & $49(43.0)$ & $11(22.4)$ & 13 & 12 & 13 & $38(77.6)$ & \\
\hline T stage & & & & & & & 0.139 \\
\hline 1 & $0(0)$ & $0(0)$ & - & - & - & $0(0)$ & \\
\hline 2 & $20(17.5)$ & $2(10)$ & 7 & 5 & 6 & $18(90)$ & \\
\hline 3 & $88(77.2)$ & $29(33)$ & 19 & 20 & 20 & $59(67.0)$ & \\
\hline 4 & $6(5.3)$ & $1(16.7)$ & 3 & 2 & 0 & $5(83.3)$ & \\
\hline TNM stage & & & & & & & 0.166 \\
\hline 1 & $13(11.4)$ & $1(7.7)$ & 4 & 2 & 6 & $12(92.3)$ & \\
\hline 2 & $46(40.4)$ & $12(26.1)$ & 14 & 12 & 8 & $34(73.9)$ & \\
\hline 3 & $46(40.4)$ & $16(34.8)$ & 9 & 10 & 11 & $30(65.2)$ & \\
\hline 4 & $9(7.9)$ & $3(33.3)$ & 2 & 3 & 1 & $6(66.7)$ & \\
\hline Differentiation & & & & & & & 0.315 \\
\hline Well & $2(1.8)$ & $1(50)$ & 1 & 0 & 0 & $1(50)$ & \\
\hline Moderate & $98(86.0)$ & $25(25.5)$ & 24 & 25 & 24 & $73(74.5)$ & \\
\hline Poor & $3(2.6)$ & $2(66.7)$ & 1 & 0 & 0 & $1(33.3)$ & \\
\hline Mucinous & $10(8.8)$ & $3(36.4)$ & 3 & 2 & 2 & $7(63.6)$ & \\
\hline Others & $1(0.9)$ & $1(100)$ & 0 & 0 & 0 & $0(0)$ & \\
\hline
\end{tabular}

Values are presented as number of cases (\%).

APC, anaphase-promoting complex; IHC, immunohistochemistry.

The results of the immunohistochemical staining are summarized in Table 2. In this study, known prognostic parameters in colon cancer, such as location, AJCC staging, T stage, tumor differentiation, Ki-67 expression, and recurrence, were confirmed to have significant impacts on patient prognosis. APC expression was not associated with $\mathrm{T}$ stage or nodal stage or with the type of differentiation between the APC 0, 1 and the APC 2, 3 groups. However, the negative expression of APC is correlated with the expression of Ki-67 (Kappa $=0.11, \mathrm{P}=0.028)$ and is correlated with lower recurrent rates $(\mathrm{P}=0.044)$ (Table 3$)$. A comparison between the strength of APC expression and the prognosis showed that a high APC expression was not associated with a favorable oncologic outcome, although the prognostic factors showing favorable outcomes were inconsistent between studies (Fig. 3).

The relationship between APC expression and oncologic outcomes has not been elucidated sufficiently. A comparison between the strength of APC expression and the 5-year overall survival rate in a Kaplan-Meier survival analyses for the APC 0, 1 and 2, 3 groups, respectively, showed significantly different 5-year 
Table 3. Correlation analysis of immunohistochemical expression of Ki-67 and APC (considering the totality of 114 patients $)(\mathrm{P}<0.05)$

\begin{tabular}{|c|c|c|c|c|c|c|c|}
\hline \multirow{3}{*}{ Variable } & \multirow{3}{*}{$\begin{array}{c}\text { No. of } \\
\text { cases } \\
\text { (\% of total) }\end{array}$} & \multicolumn{5}{|c|}{ APC expression IHC grade } & \multirow{3}{*}{ P-value } \\
\hline & & \multirow{2}{*}{$\frac{\text { Negative }}{0}$} & \multicolumn{4}{|c|}{ Positive } & \\
\hline & & & 1 & 2 & 3 & All & \\
\hline Ki-67 expression & & & & & & & 0.028 \\
\hline Grade 0 & $7(6.1)$ & $5(71.4)$ & 1 & 0 & 1 & $2(28.6)$ & \\
\hline Grade 1 & $35(30.7)$ & $8(22.9)$ & 12 & 9 & 6 & $27(77.1)$ & \\
\hline Grade 2 & $27(23.7)$ & $10(37.0)$ & 6 & 6 & 5 & $17(63.0)$ & \\
\hline Grade 3 & 45 (39.5) & $9(20.0)$ & 10 & 12 & 14 & $36(80.0)$ & \\
\hline Disease recurrence & & & & & & & 0.044 \\
\hline No & $48(42.1)$ & $8(16.7)$ & 12 & 12 & 16 & 40 (83.3) & \\
\hline Yes & $32(28.1)$ & $9(28.1)$ & 10 & 7 & 6 & 23 (71.9) & \\
\hline
\end{tabular}

Values are presented as number of cases (\%).

APC, anaphase-promoting complex; IHC, immunohistochemistry.

overall survival rates (APC 0,1 groups: $57 \%$ vs. APC 2, 3 groups: $74 \%, \mathrm{P}=0.042$ ) (Fig. 3). Although studies have investigated the survival of patients with a colorectal adenocarcinoma according to APC expression, data are insufficient to draw any conclusions.

\section{DISCUSSION}

Postoperative evaluation of the prognosis of patients with colorectal cancer is very important. After surgery, several genetic factors, including p53, CD95, PINCH, Ki-67, thymidylate synthase, and high S-phase fraction, are studied $[10,12]$ in patients with colorectal cancer. However, whether or not the regulation of APC is abnormal in patients with colorectal cancer is unknown. Chromosome instability through abnormal mitotic progression has been reported to play a critical role in tumor malignancy $[13,14]$. Therefore, the dysregulation of APC activation, which probably perturbs mitotic progression, may affect malignant transformation or tumor progression. Moreover, the finding that APC is required for the G2 and the mitotic checkpoints suggests that malignant transformation can be caused by chromosome instability through the dysregulation of APC activation [15]. Recently, Wang et al. [16] reported a genetic alteration in APC6 and APC8 in human colon cancer cells and suggested their involvement in colon carcinogenesis.

During mitosis, various APC molecules, such as ubiquitin ligase (E3), which is composed of tetratricopeptide repeat proteins (APC3, 5, 6, 7, and 8), cullin homolog (APC2), and ring-H2 finger domain (APC11), have been identified in yeast. Ubiquitin ligase (E3) is bound to the cdc20 and the cdh1 that regulate the cell cycle, and it promotes the transition from the metaphase to the anaphase [17]. Among them various molecules, APC7 in only the vertebral expressions of nondegradable securin mutants or the overexpressions of wild-type securin blocks sister chromatid separation [18], and, more importantly, the deletion of securin in
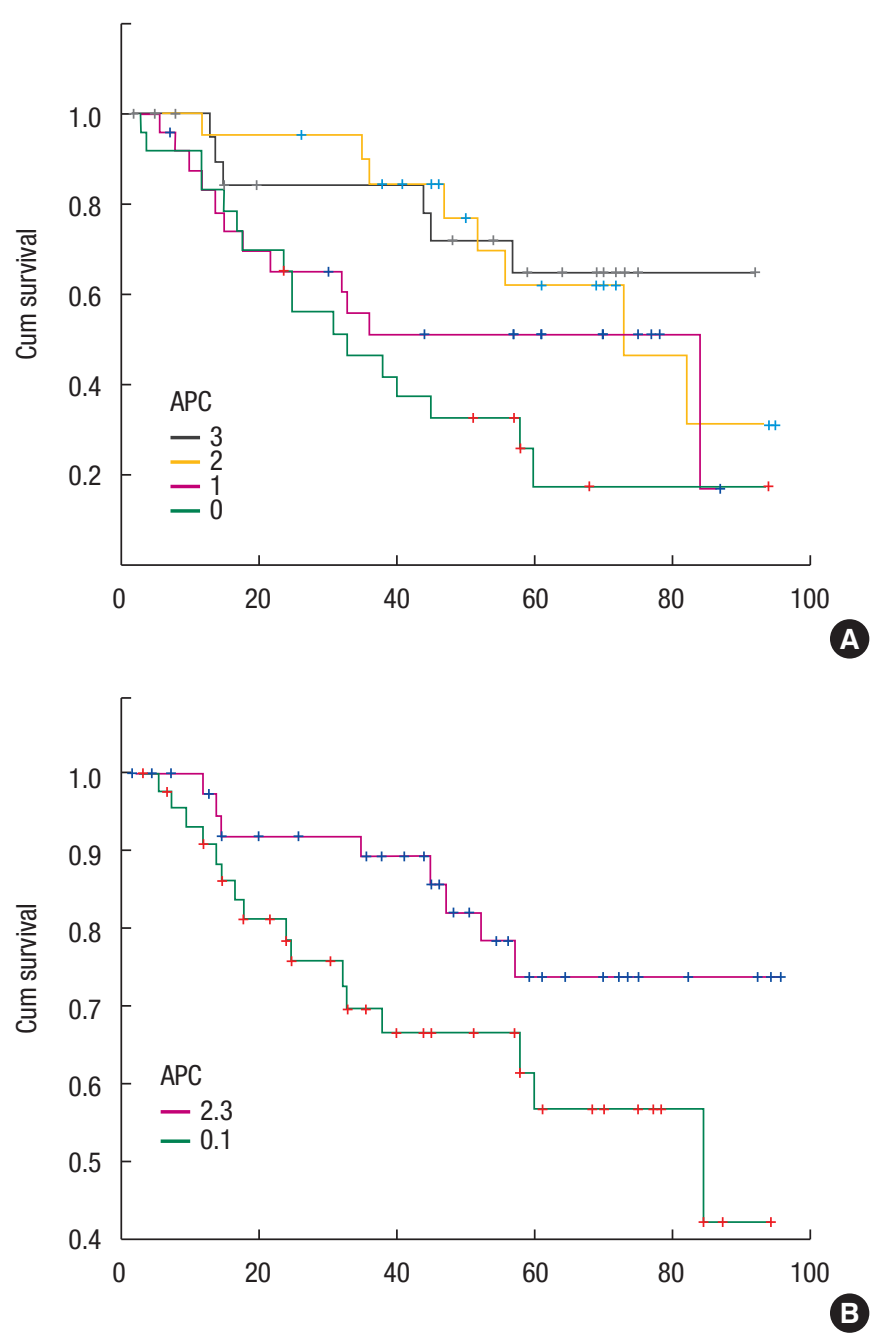

Fig. 3. Five-year overall survival rate from the Kaplan-Meier survival analysis according to the anaphase-promoting complex (APC) 0,1 groups $(57 \%)$ and the APC 2, 3 groups $(74 \%)(\mathrm{P}=0.042)$. (A) Relation between the strength of APC and the prognosis. (B) Picture showing that negative and the grade 1 could be made into a group and that grades 2 and 3 could grouped together as well, which allows the prognosis comparison to be carried out.

budding yeast allows sister chromatid separation in the absence of APC participation. These results imply that APC's essential role in initiating the anaphase must be the destruction of securin. Polyubiquitin chains are added to securin by an E3 ubiquitin ligase known as anaphase-promoting complex or cyclosome. If separase is inhibited, chromatids cannot be separated; consequently, DNA aneuploidy occurs, which is associated with human cancer and with poor clinical 5-year outcomes in patients with colorectal cancers [19]. Thus, whether dysregulation of APC is related to clinical parameters in various human colorectal cancers has yet to be determined. Therefore, we decided to investigate the expression of APC7 in 114 colorectal cancers and to inquire into the re- 
lationship between the expression of APC7 and the clinicopathologic parameters.

According to this study, the degree of APC7 expression was not related to age, sex, or the tumor's location. Also, the degree of differentiation was not related to the type of tumor. The possibility of a colorectal carcinoma might be increased if APC is not expressed or if the amount of APC is decreased. In either case, the survival rate of the patients declines. The results of research efforts studying the relationship between the occurrence of a carcinoma of the large intestine and aneuploidy have been published for a long time, and as a result of such studies, DNA aneuploidy is now known to be associated with a poor clinical 5-year outcome in patients with colorectal cancer [19]. A decrease in the amount of APC may gradually lead to a carcinoma of the large intestine and may cause harm after recovery. An assessment of tumor cell proliferation may predict tumor behavior, and the typical correlative protein that can be used in paraffin embedding as an immunohistochemical staining agent is $\mathrm{Ki}-67$. Ki-67 is a monoclonal antibody that plays an important, though not entirely characterized, role in cell proliferation. Ki-67 is a tumor proliferative index, which is expressed in the G1, S and G2-M phases, but not in the G0 phase. This protein is expressed in the nuclei of all proliferating cells, but is absent in resting cells [10]. The correlation between the expression of Ki-67 and prognosis has been found in a number of cancer studies. It is a unicell group antibody as the core part of the cell cycle coming from a Hodgkin lymphoma, and it is expressed during all phases of cell division except the stationary phase [1]. Statistically, the lower the index of Ki-67 is, the more the expression of APC increases, which suggests an inverse relation between Ki-67 and APC. This research focused on the APC protein and later on the investigation of whether reverse transcription-polymerase chain reaction, real-time polymerase chain reaction, enzyme-linked immunosorbent assay, Western blotting, flow-cytometric analyses revealed significant information about the prognosis.

The current study has some limitations. First, it was not a randomized controlled study; thus, the results could have been affected by potential selection bias. Second, the sample size (114 patients) was not estimated because tumor tissues were retrospectively recruited, so a sample-size estimate could not be performed. Finally, the sample size was relatively small. In conclusion, a positive APC expression level was found to be associated with lower recurrence and better overall survival. Thus, further study of APC's role in controlling alterations in the cell cycle and its association with the invasiveness and the proliferation of tumor cells is needed.

\section{CONFLICT OF INTEREST}

No potential conflict of interest relevant to this article was reported.

\section{ACKNOWLEDGMENTS}

This study was supported a research grant from Yonsei University Wonju College of Medicine (YUWCM 2015-001), Wonju, Republic of Korea.

\section{REFERENCES}

1. Peters JM. The anaphase-promoting complex: proteolysis in mitosis and beyond. Mol Cell 2002;9:931-43.

2. Zachariae W, Nasmyth K. Whose end is destruction: cell division and the anaphase-promoting complex. Genes Dev 1999;13:203958 .

3. Vodermaier HC, Gieffers C, Maurer-Stroh S, Eisenhaber F, Peters JM. TPR subunits of the anaphase-promoting complex mediate binding to the activator protein CDH1. Curr Biol 2003;13:145968.

4. Hanahan D, Weinberg RA. The hallmarks of cancer. Cell 2000; 100:57-70.

5. Lengauer C, Kinzler KW, Vogelstein B. Genetic instabilities in human cancers. Nature 1998;396:643-9.

6. Wang X, Jin DY, Ng RW, Feng H, Wong YC, Cheung AL, et al. Significance of MAD2 expression to mitotic checkpoint control in ovarian cancer cells. Cancer Res 2002;62:1662-8.

7. Li Y, Benezra R. Identification of a human mitotic checkpoint gene: hsMAD2. Science 1996;274:246-8.

8. Pihan GA, Doxsey SJ. The mitotic machinery as a source of genetic instability in cancer. Semin Cancer Biol 1999;9:289-302.

9. Sudakin V, Chan GK, Yen TJ. Checkpoint inhibition of the APC/ $\mathrm{C}$ in HeLa cells is mediated by a complex of BUBR1, BUB3, CDC20, and MAD2. J Cell Biol 2001;154:925-36.

10. Ishida $H$, Miwa $H$, Tatsuta M, Masutani S, Imamura H, Shimizu J, et al. Ki-67 and CEA expression as prognostic markers in Dukes' C colorectal cancer. Cancer Lett 2004;207:109-15.

11. Park KH, Choi SE, Eom M, Kang Y. Downregulation of the anaphase-promoting complex (APC)7 in invasive ductal carcinomas of the breast and its clinicopathologic relationships. Breast Cancer Res 2005;7:R238-47.

12. Sträter J, Hinz U, Hasel C, Bhanot U, Mechtersheimer G, Lehnert $\mathrm{T}$, et al. Impaired CD95 expression predisposes for recurrence in curatively resected colon carcinoma: clinical evidence for immunoselection and CD95L mediated control of minimal residual disease. Gut 2005;54:661-5.

13. Duesberg P, Li R. Multistep carcinogenesis: a chain reaction of aneuploidizations. Cell Cycle 2003;2:202-10.

14. Sen S. Aneuploidy and cancer. Curr Opin Oncol 2000;12:82-8.

15. Sudo T, Ota Y, Kotani S, Nakao M, Takami Y, Takeda S, et al. Activation of Cdh1-dependent APC is required for G1 cell cycle arrest and DNA damage-induced G2 checkpoint in vertebrate cells. EMBO J 2001;20:6499-508.

16. Wang Q, Moyret-Lalle C, Couzon F, Surbiguet-Clippe C, Saurin JC, Lorca T, et al. Alterations of anaphase-promoting complex 
genes in human colon cancer cells. Oncogene 2003;22:1486-90.

17. Hershko A, Ciechanover A. The ubiquitin system. Annu Rev Biochem 1998;67:425-79.

18. Alberts B, Bray D, Lewis J, Raff M, Roberts K. Molecular biology of the cell. 3th ed. New York: Garland Publishing Inc.; 1994.
19. Bazan V, Migliavacca M, Zanna I, Tubiolo C, Corsale S, Calò V, et al. DNA ploidy and S-phase fraction, but not p53 or NM23-H1 expression, predict outcome in colorectal cancer patients. Result of a 5-year prospective study. J Cancer Res Clin Oncol 2002;128: 650-8. 